\title{
Position Control of a Solenoid Based Linearly Movable Armature System using Robust Control Technique
}

\author{
Mustefa Jibril ${ }^{1}$, Messay Tadese ${ }^{2}$, Eliyas Alemayehu Tadese ${ }^{3}$ \\ ${ }^{1}$ Msc, School of Electrical \& Computer Engineering, Dire Dawa Institute of Technology, Dire Dawa, \\ Ethiopia \\ ${ }^{2}$ Msc, School of Electrical \& Computer Engineering, Dire Dawa Institute of Technology, Dire Dawa, \\ Ethiopia \\ ${ }^{3}$ Msc, Faculty of Electrical \& Computer Engineering, Jimma Institute of Technology, Jimma, Ethiopia
}

\begin{abstract}
In this paper, a solenoid based linearly movable armature system is designed using robust control theory in order to improve the performance of the system. Reference track method is the best performance analysis for position control systems. Among the robust controllers, H infinity mixedsensitivity and Mixed H $2 / \mathrm{H} \infty$ with Regional Pole Placement Controllers are used to improve the performance of the system. Comparison of the proposed controllers for tracking a reference displacement signals (step and sine wave) and a promising simulation result have been obtained.
\end{abstract}

Keywords: solenoid; robust control theory; H infinity mixed-sensitivity; Mixed H 2 /Hळ

\section{Introduction}

A solenoid valve is an electromechanically operated valve. Solenoid valves fluctuate within the traits of the electric contemporary they use, the energy of the magnetic area they generate, the mechanism they use to alter the fluid, and the kind and traits of fluid they control. The mechanism varies from linear action, plunger-kind actuators to pivoted-armature actuators and rocker actuators. The valve can use a -port layout to regulate a glide or use a 3 or more port design to switch flows between ports. Multiple solenoid valves may be positioned collectively on a manifold. Solenoid valves are the most frequently used manipulate factors in fluidics. Their duties are to close off, launch, dose, distribute or mix fluids. They are discovered in many utility areas. Solenoids offer fast and safe switching, excessive reliability, lengthy carrier life, desirable medium compatibility of the substances used, low manipulate strength and compact design. In a few solenoid valves the solenoid acts at once on the fundamental valve. Others use a small, complete solenoid valve, called a pilot, to actuate a larger valve. While the $2 \mathrm{~d}$ type is genuinely a solenoid valve mixed with a pneumatically actuated valve, they may be offered and packaged as a unmarried unit referred to as a solenoid valve. Piloted valves require a lot less energy to control, however they are highly slower. Piloted solenoids normally want full power always to open and live open, wherein a direct acting solenoid may also simplest want full energy for a quick period of time to open it, and handiest low power to maintain it. In this paper, solenoid based linearly movable armature system is designed and controlled using robust control technique and the performance of the system is analyzed based on track a reference approach. 


\section{Mathematical Model}

The dynamics of the solenoid based linearly movable armature system is shown in Figure 1.

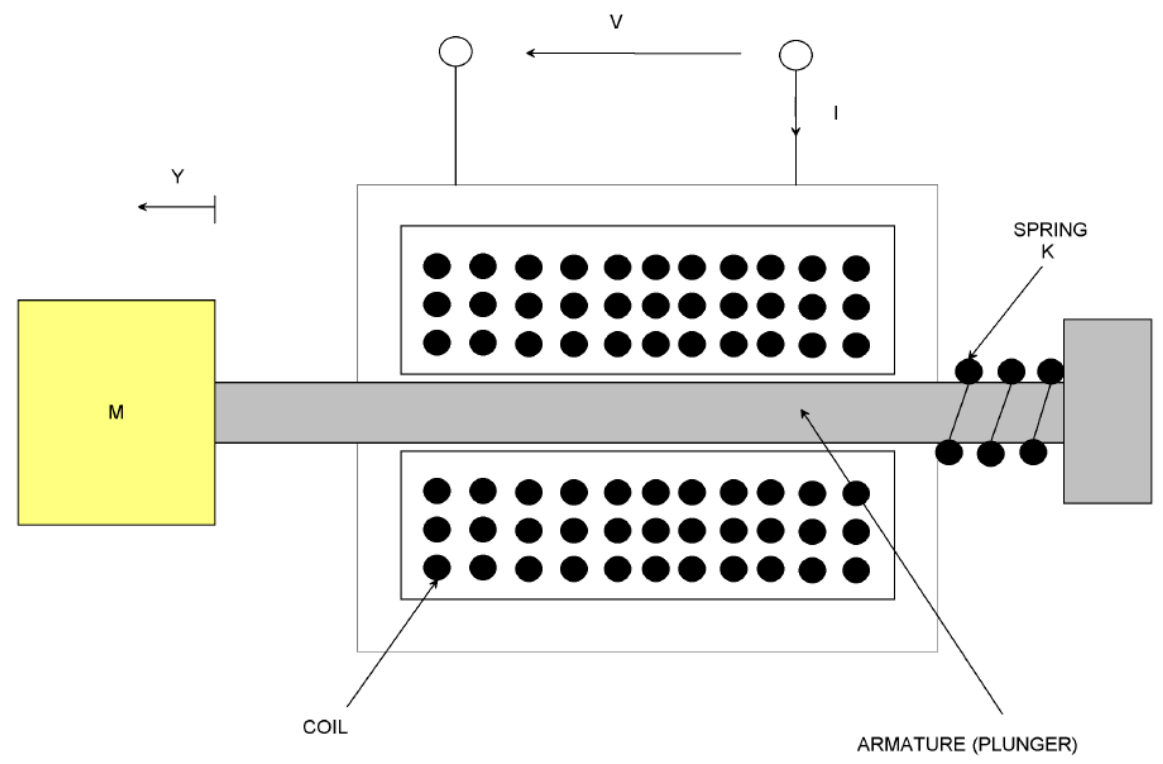

Figure 1 Solenoid based linearly movable armature system

The magnetic flux linkage can be described by

$$
\Psi=\Psi_{0}+L_{d} I+L_{C} Y
$$

Where

$\Psi_{0}$ Initial magnetic flux linkage

$L_{d}$ Differential inductance

$L_{C}$ Coil inductance

The magnet circuit equation will be

$$
V(t)=R I(t)+L_{d} \frac{d I(t)}{d t}+L_{C} \frac{d Y}{d t}
$$

The force exerted by the magnetic circuit to the mass become

$$
F_{m}(t)=L_{C} I(t)
$$

The spring-mass system model equation is given by

$$
F_{m}(t)=M \&(t)+b Y^{\&}(t)+K Y(t)
$$

Upon selecting the voltage $V(t)$ as the variable to be manipulated and the linear displacement as the variable to be controlled, substituting Equation (3) in to Equation (4) and in to Equation (2) and taking the Laplace transform results the transfer function 


$$
G(s)=\frac{Y(s)}{V(s)}=\frac{\frac{L_{C}}{K R}}{\frac{M L_{d}}{K R} s^{3}+\left(\frac{M}{K}+\frac{b L_{d}}{K R}\right) s^{2}+\left(\frac{b}{K}+\frac{L_{d}}{R}+\frac{L_{C}{ }^{2}}{K R}\right) s+1}
$$

The system parameters are shown in Table 1 below

Table 1 System parameters

\begin{tabular}{|c|c|c|c|}
\hline No & Parameters & Symbols & Value \\
\hline 1 & Mass of the body & $M$ & $3 \mathrm{Kg}$ \\
\hline 2 & Resistance of the coil & $R$ & $10 \mathrm{ohm}$ \\
\hline 3 & Differential inductance & $L_{d}$ & $5 \mathrm{H}$ \\
\hline 4 & Coil inductance & $L_{C}$ & $10 \mathrm{H}$ \\
\hline 5 & Damping friction & $b$ & $3 \mathrm{~N}-\mathrm{s} / \mathrm{m}$ \\
\hline 6 & Spring stiffness & $K$ & $6 \mathrm{~N} / \mathrm{m}$ \\
\hline
\end{tabular}

The transfer function of the system numerically become

$$
G(s)=\frac{Y(s)}{V(s)}=\frac{0.1667}{0.25 s^{3}+0.75 s^{2}+2.667 s+1}
$$

The state space model become

$$
\begin{aligned}
& x=\left(\begin{array}{ccc}
-3 & -10.667 & -4 \\
1 & 0 & 0 \\
0 & 1 & 0
\end{array}\right) x+\left(\begin{array}{l}
1 \\
0 \\
0
\end{array}\right) u \\
& y=\left(\begin{array}{lll}
0 & 0 & 0.6667
\end{array}\right)
\end{aligned}
$$

\section{Proposed Controllers Design}

\subsection{H infinity Mixed-Sensitivity Controller Design}

$\mathrm{H}$ infinity mixed-sensitivity evaluates a controller $\mathrm{K}$ that minimizes the $\mathrm{H}$ infinity norm of the closed-loop transfer function the mixed weighted sensitivity

$$
T_{y 1 u 1 \triangleq}\left[\begin{array}{l}
W_{1} S \\
W_{2} R \\
W_{3} T
\end{array}\right]
$$

Where $\mathrm{S}$ and $\mathrm{T}$ are referred to as the sensitivity and complementary sensitivity, respectively. $\mathrm{R}$ measures the manipulate effort. The again controller $\mathrm{K}$ is such that $\mathrm{S}, \mathrm{R}$, and $\mathrm{T}$ fulfill the following loop-shaping inequalities: 


$$
\begin{aligned}
& \bar{\sigma}(S(j \omega)) \leq \gamma \underline{\sigma}\left(W_{1}^{-1}(j \omega)\right) \\
& \bar{\sigma}(R(j \omega)) \leq \gamma \underline{\sigma}\left(W_{2}^{-1}(j \omega)\right) \\
& \bar{\sigma}(T(j \omega)) \leq \gamma \underline{\sigma}\left(W_{3}^{-1}(j \omega)\right)
\end{aligned}
$$

Where $\gamma=$ GAM. Thus, the inverses of $\mathrm{W} 1$ and $\mathrm{W} 3$ determine the shapes of sensitivity $\mathrm{S}$ and complementary sensitivity T. Typically, you choose a W1 this is large within the desired manipulate bandwidth to acquire accurate disturbance attenuation (i.e., performance). Similarly, you commonly pick a W3 that is large outside the manage bandwidth, which facilitates to make sure precise stability margin. The solenoid based linearly movable armature system with H infinity mixed sensitivity controller block diagram is shown in Figure 2 below.

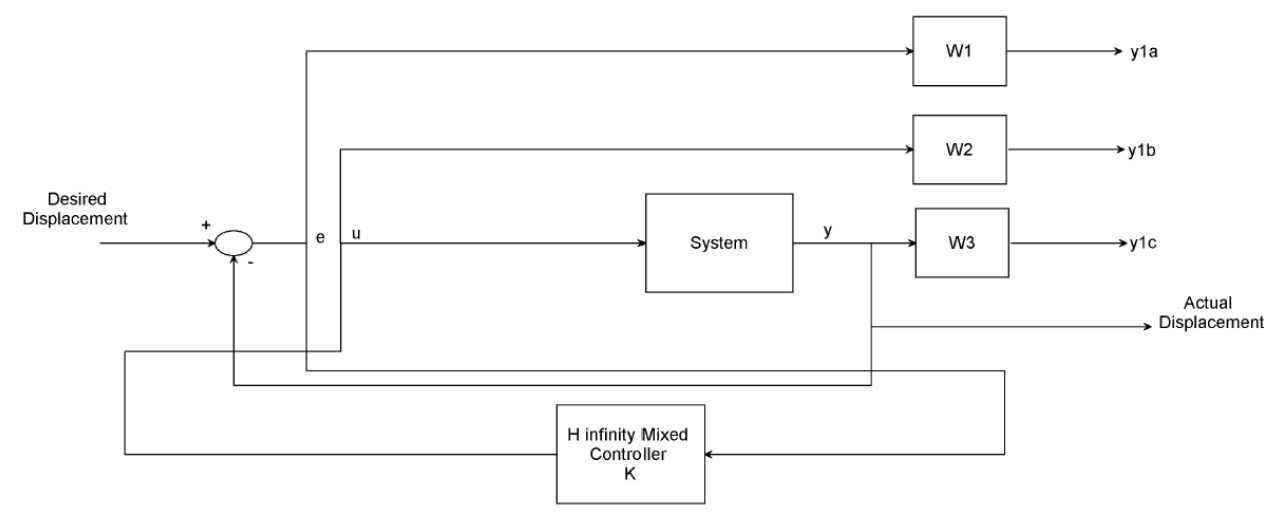

Figure 2 Solenoid based linearly movable armature system with $\mathrm{H}$ infinity mixed sensitivity controller block diagram

Here in this system we choose the three weighting functions W1, W2 and W3 as

$$
\begin{aligned}
& W_{1}=\frac{1}{s+12} \\
& W_{2}=\frac{1}{s+11} \\
& W_{3}=\frac{1}{s+8}
\end{aligned}
$$

The Controller transfer function is

$$
K_{\text {mixed }}=10 \frac{s^{2}+107 s+70.5}{s^{2}+95 s+1}
$$

\subsection{Mixed H 2 /Hळ with Regional Pole Placement Controller design}

The mixed $\mathrm{H} 2 / \mathrm{H} \infty$ control hassle is to reduce the $\mathrm{H} 2$ norm of overall state remarks gains $\mathrm{K}$ such that what also satisfies the $\mathrm{H} \infty$ norm constraint. Mixed $\mathrm{H} 2 / \mathrm{H} \infty$ synthesis with regional pole 
placement is one example of multi-objective layout addressed by means of the LMI. The manipulate problem is sketched in Figure 3. The output channel $\mathrm{z}$ is associated with the $\mathrm{H} \infty$ overall performance at the same time as the channel $\mathrm{z} 2$ is associated with the $\mathrm{H} 2$ overall performance.

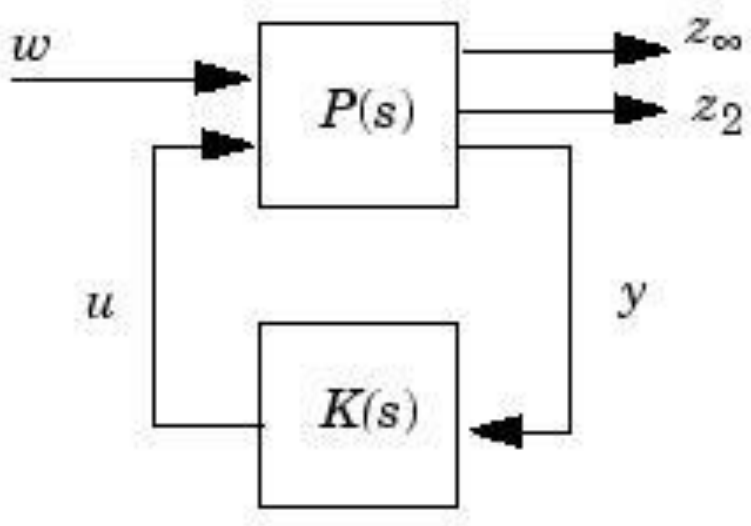

Figure 3 Mixed H 2 /Hळ configuration

The LMI region for the pole placement is determined the use of the command lmireg and we select the $1 / 2$ plane region and the output region is

$$
2.0000+1.0000 i \text { and } 1.0000+0.0000 i
$$

And we use this region for the mixed $\mathrm{H} 2 / \mathrm{H} \infty$ controller synthesis.

The transfer function of the controller is

$$
K_{\text {mixed }}=12 \frac{s^{2}+118 s+100}{s^{2}+100 s+1}
$$

\section{Result and Discussion}

In this section, the Simulink model design and simulation of the solenoid based linearly movable armature system using $\mathrm{H}$ infinity mixed-sensitivity and Mixed $\mathrm{H} 2 / \mathrm{H} \infty$ with regional pole placement controllers by comparing the two proposed controllers for tracking the step and sine wave references displacement signals.

\subsection{Comparison of the proposed controllers for tracking the step reference displacement}

The Simulink model of the solenoid based linearly movable armature system using $\mathrm{H}$ infinity mixed-sensitivity and mixed $\mathrm{H} 2 / \mathrm{H} \infty$ with regional pole placement controllers for tracking the step references displacement signal is shown in Figure 4 below. 


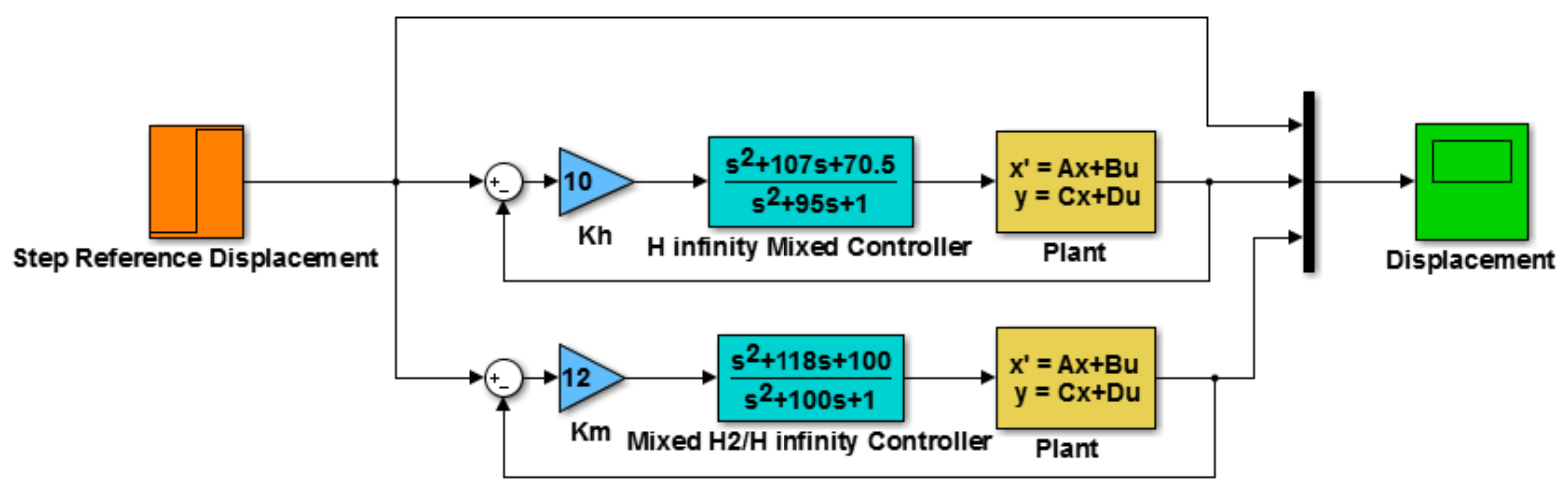

Figure 4 Simulink model of the solenoid based linearly movable armature system using $\mathrm{H}$ infinity mixed-sensitivity and mixed $\mathrm{H} 2 / \mathrm{H} \infty$ with regional pole placement controllers for tracking the step references displacement signal

The solenoid based linearly movable armature system performance for the proposed controllers using a step reference (step change from 0 to $6 \mathrm{~m}$ ) of the displacement output simulation is shown in Figure 5 below.

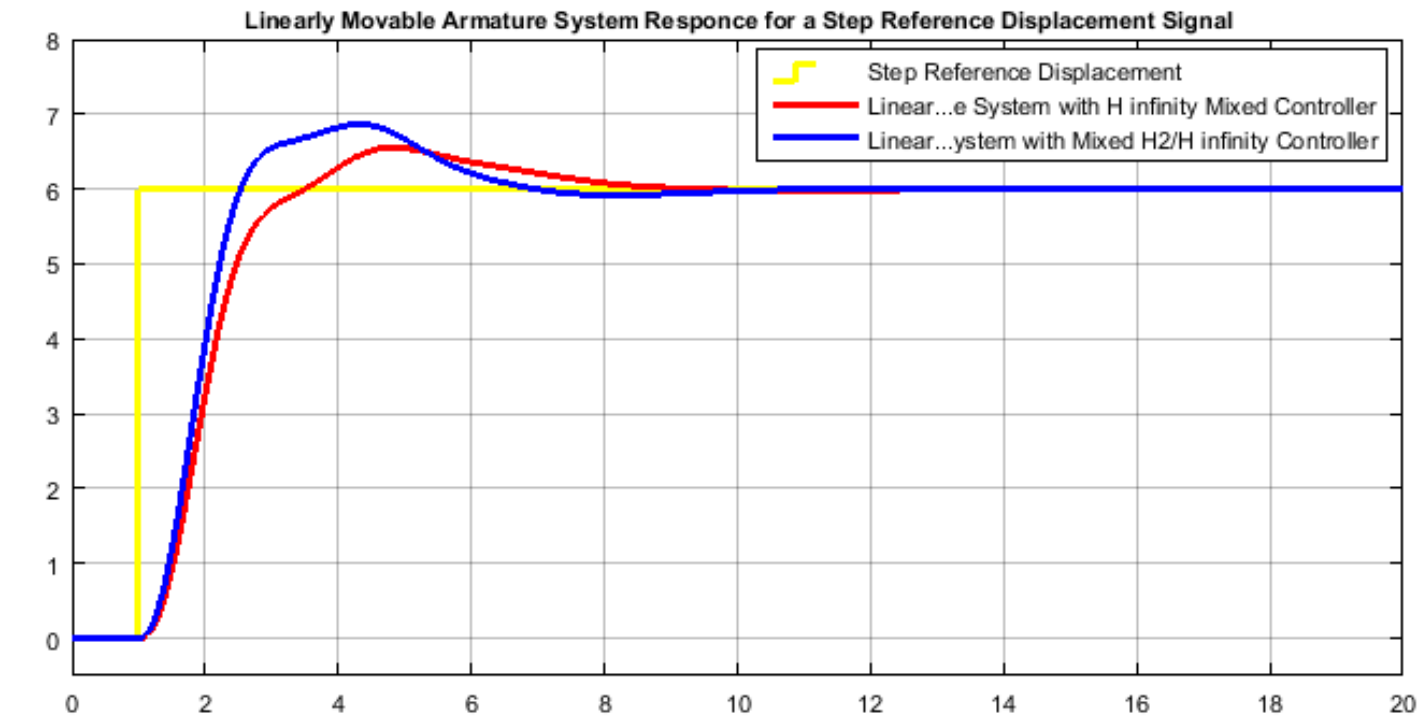

Time offset: 0

Figure 5 Simulation result for a step reference input

The data of the rise time, percentage overshoot, settling time and peak value is shown in Table 1.

Table 1 Step response data

\begin{tabular}{|c|c|c|c|}
\hline No & Performance Data & H infinity mixed-sensitivity & Mixed H 2/Hळ \\
\hline 1 & Rise time & $2 \mathrm{sec}$ & $1.9 \mathrm{sec}$ \\
\hline 2 & Per. overshoot & $8.33 \%$ & $15 \%$ \\
\hline 3 & Settling time & $10 \mathrm{sec}$ & $13 \mathrm{sec}$ \\
\hline
\end{tabular}




\begin{tabular}{|c|c|c|c|}
\hline 4 & Peak value & $6.9 \mathrm{~m}$ & $6.5 \mathrm{~m}$ \\
\hline
\end{tabular}

\subsection{Comparison of the proposed controllers for tracking the Sine Wave speed reference}

The Simulink model of the solenoid based linearly movable armature system using $\mathrm{H}$ infinity mixed-sensitivity and mixed $\mathrm{H} 2 / \mathrm{H} \infty$ with regional pole placement controllers for tracking the sine wave references displacement signal is shown in Figure 6 below.

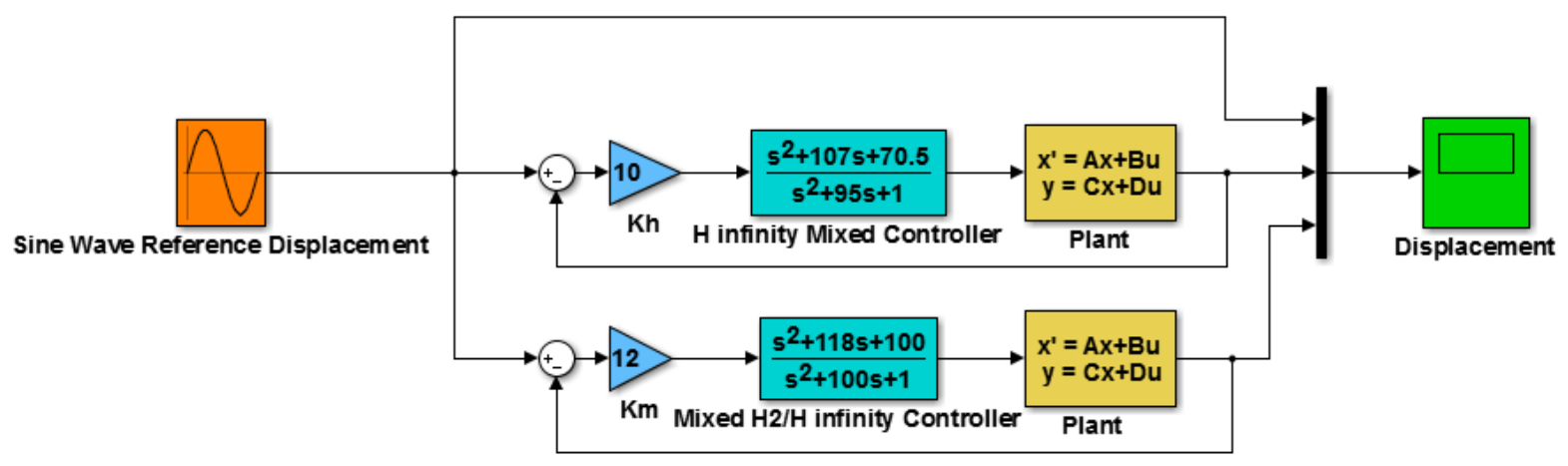

Figure 6 Simulink model of the solenoid based linearly movable armature system using $\mathrm{H}$ infinity mixed-sensitivity and mixed $\mathrm{H} 2 / \mathrm{H} \infty$ with regional pole placement controllers for tracking the sine wave references displacement signal

The solenoid based linearly movable armature system performance for the proposed controllers using a sine wave reference (displacement moving in the forward and reverse with $6 \mathrm{~m}$ ) of the displacement output simulation is shown in Figure 7 below.

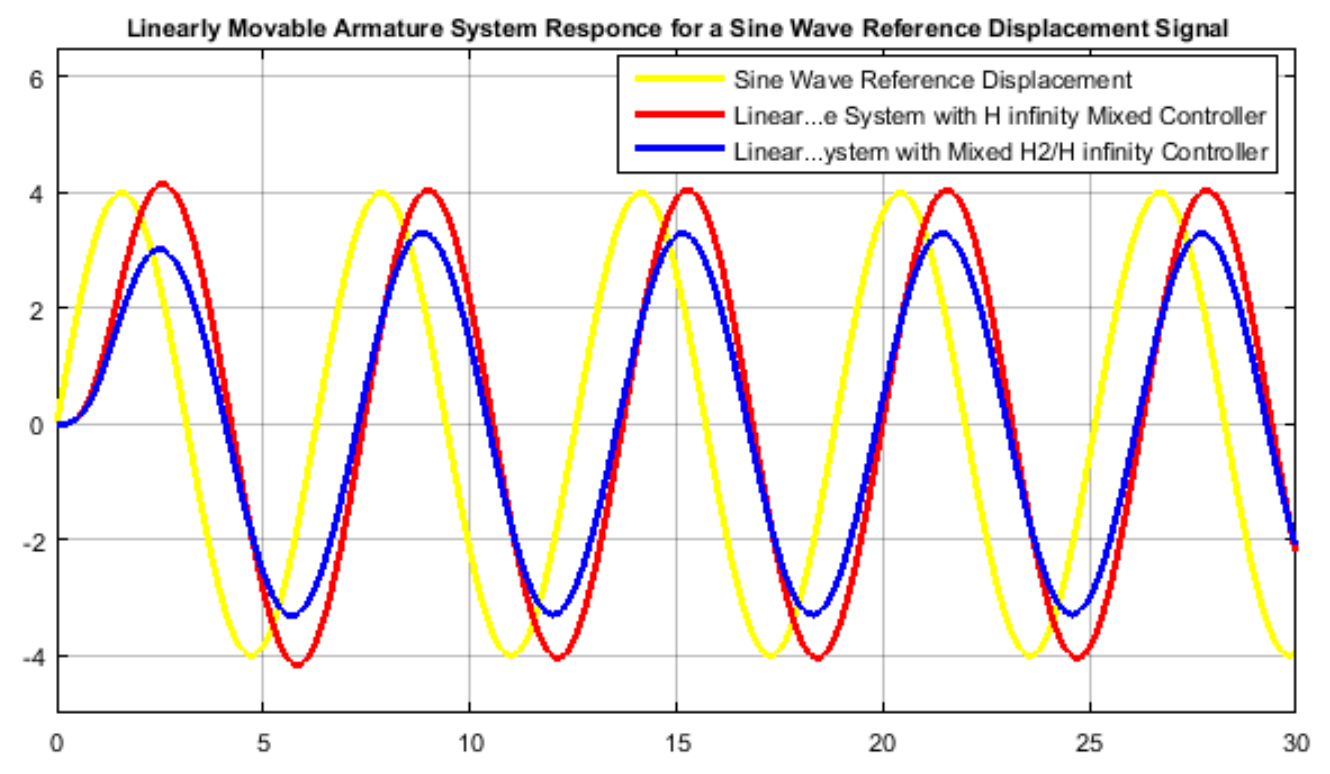

Time offset: 0 
Figure 7 Simulation result for a sine wave reference input

The simulation result shows that the solenoid based linearly movable armature system with $\mathrm{H}$ infinity mixed-sensitivity controller track the reference speed better than the solenoid based linearly movable armature system with mixed $\mathrm{H} 2 / \mathrm{H} \infty$ with regional pole placement controller.

\section{Conclusion}

In this paper, modelling, design and control of the solenoid based linearly movable armature system using $\mathrm{H}$ infinity mixed-sensitivity and mixed $\mathrm{H} 2 / \mathrm{H} \infty$ with regional pole placement controllers have been successfully done with the aid of Matlab/Simulink Toolbox. The proposed controllers improved the performance of the position controlling mechanism and comparison of the system with the two controllers for tracking a reference displacement signals (step and sine wave) is done. The solenoid based linearly movable armature system using $\mathrm{H}$ infinity mixedsensitivity controller has better percentage overshoot and settling time in the step reference displacement signal and better tracking the input signal in the sine wave reference displacement signal than the solenoid based linearly movable armature system with mixed $\mathrm{H} 2 / \mathrm{H} \infty$ with regional pole placement controller.

\section{Reference}

[1]. Yongho Jeon et al. "Pi Controller Design for Stable Solenoid Valve System Using the Scheduling of Control Gain" International Journal of Innovative Technology and Exploring Engineering (IJITEE), Vol. 8, Issue 4S2, pp. 9-11, 2019.

[2].M. F Badr "Modelling and Simulation of a Controlled Solenoid" IOP Conference Series: Materials Science and Engineering, Vol. 433, 2018.

[3].Hao T. et al. "Coil Inductance Model Based Solenoid ON/OFF Valve Spool Displacement Sensing via Laser Calibration” Sensors (Basel), Vol. 18, No. 12, 2018.

[4].Hua Z. "The Characteristic Improvement of Electromagnetic Proportional Directional Control Valve" Journal of Control Science and Engineering, Vol. 2018, 8 pages, 2018.

[5]. Munaf F. et al. "Position Control of the Pneumatic Actuator Employing ON/OFF Solenoids Valve" International Journal of Mechanical \& Mechatronics Engineering, Vol. 17, Issue 2, pp. 29-37, 2017.

[6].Jae Hak L. et al. "Control of Spool Position of ON/OFF Solenoid Operated Hydraulic Valve by Sliding Mode Controller" Journal of Mechanical Science and Technology, Vol. 29, pp. 5395-5408, 2015. 